\title{
A ATIVIDADE FÍSICA E OS PRINCIPAIS EFEITOS NO CONTROLE DA DIABETES E OBESIDADE NA GESTAÇÃO
}

DOI: 10.48140/digitaleditora.2020.002.15

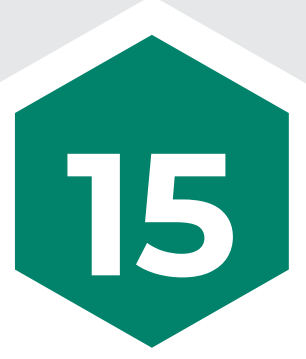

RESUMO

Objetivos: Discutir sobre a Atividade física e os principais efeitos no controle da diabetes e obesidade na gestação, e os principais benefícios obtidos através na prescrição de diferentes tipos de atividade física em gestantes.

Métodos: Para a realização desta pesquisa foram realizados levantamentos bibliográficos de artigos científicos achados nas bases de dado Pubmed, The Scientific Electronic Library Online - (SCIELO), referentes à temática do trabalho.

Resultados: Os resultados vistos no estudo relatam que gestantes com comorbidades, como diabetes e obesidade que fizeram exercícios físicos com acompanhamento dentro dos níveis de intensidade indicado, tiveram êxitos que não comprometeram o período gestacional nem o feto.

Conclusão: A atividade física durante a gestação traz inúmeros benefícios para a mãe e para o feto, desde que sejam tomados os devidos cuidados quanto ao tipo, duração e intensidade dos exercícios, respeitando as contraindicações e patologias associadas com acompanhamento profissional e indicação médica de maneira individualizada.
Ailton Silva do Nascimento

Graduando em Educação Física pela AESPI - Ensino Superior do Piauí

Teresina - Piauí

iD https://orcid.org/0000-

iD 0001-9157-9916

Pedro Victor Ramalho de

Macêdo

Graduando em Educação Física pela AESPI - Ensino Superior do Piauí

Teresina - Piauí

iD https://orcid.org/00000002-2190-0871

\section{Mário Sérgio de Paiva Dias}

Profissional de Educação Física, Especialista e Professor Auxiliar da AESPI - Ensino Superior do Piauí Teresina - Piauí

in https://orcid.org/0000-

ID 0003-0284-2140 


\section{PHYSICAL ACTIVITY AND THE MAIN EFFECTS ON DIABETES CONTROL AND OBESITY IN PREGNANCY}

DOI: 10.48140/digitaleditora.2020.002.15

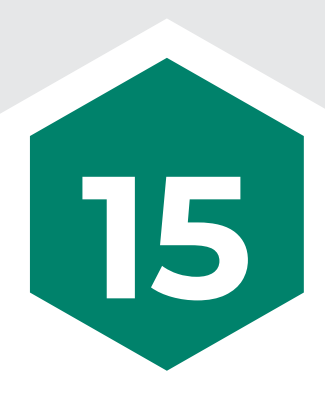

\section{ABSTRACT}

Recebido em: 10/12/2020

Aprovado em: 28/12/2020

Conflito de Interesse: não

Suporte Financeiro: não houve

Objectives: To discuss physical activity and the main effects in the control of diabetes and obesity in pregnancy, and the main benefits obtained through the prescription of different types of physical activity in pregnant women.

Methods: To carry out this research, bibliographical surveys of scientific articles found in the Pubmed databases, The Scientific Electronic Library Online - (SCIELO), referring to the theme of the work, were carried out.

Results: The results seen in the study report that pregnant women with comorbidities, such as diabetes and obesity that physical exercise with monitoring within the indicated intensity levels, had successes that do not compromise the gestational period or the fetus.

Conclusion: Physical activity during pregnancy brings countless benefits for the mother and the fetus, provided that due care is taken as to the type, duration and intensity of the exercises, respecting the contraindications and pathologies associated with professional monitoring and medical indication of individualized way. 


\section{(8) \\ INTRODUÇÃO}

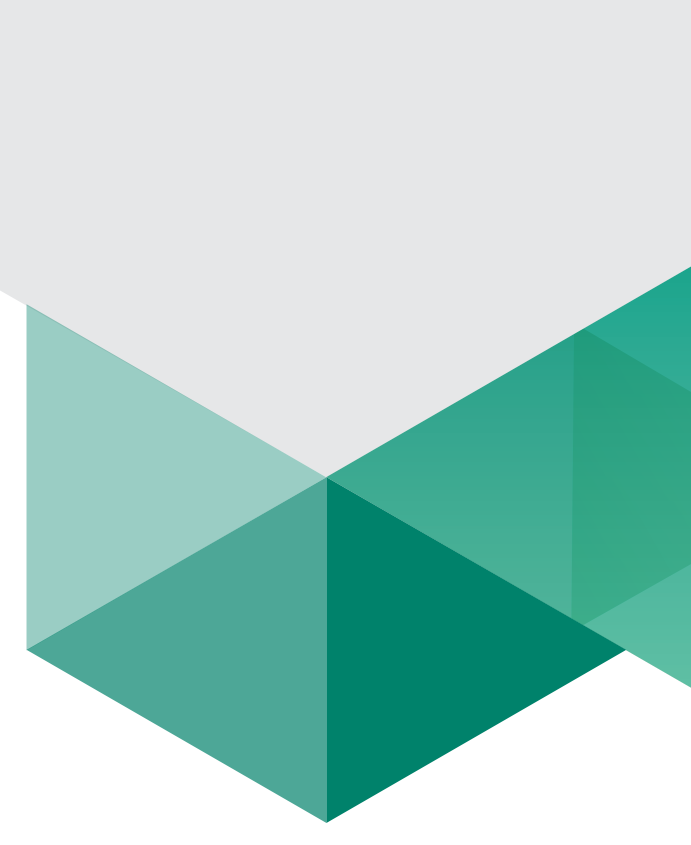

Durante a gestação a mulher sofre diversas alterações biológicas que podem ser facilmente notadas, variando de mulher para mulher dependendo também do seu tempo de gestação (SILVA et al, 2015 citados por QUEIROGA; CARRIEL; PERUCHI, 2018). Mulheres grávidas com diabetes, ou obesas são suscetíveis a elevadas modificações, com algumas consequências que podem resultar em muitas dores e limitações nas atividades diárias, uma vez que essas transformações trazem consequências em todo o sistema do corpo.

A prática de atividades físicas em gestantes com essas comorbidades, devem ser praticadas com alguns cuidados para se obter os devidos benefícios. É de total importância que as fases da gestação sejam respeitadas, e a intensidade das atividades físicas se ajustem a essas fases. Vários tipos de exercícios podem ser realizados com gestantes, como a musculação com cargas leves e exercícios aeróbicos leves. As práticas desses exercícios trazem mais benefícios que malefícios para gestante, resultando em uma gestação mais tranquila, além de uma melhor recuperação pós-parto. As grávidas saudáveis devem ser encorajadas a praticarem exercícios físicos durante todo o processo de gestação, no entanto as que possuem algum tipo de alteração, devem fazer exercícios específicos que não tragam riscos à gestante e ao bebê. Atividades Físicas podem ser definidas como um conjunto de ações que um indivíduo ou grupo de pessoas praticam envolvendo gasto de energia e alterações do organismo, por meio de movimentos corporais (SOARES D.S.C., SOARES J.J., GRAUP S., STREB A.R., 2018). É importante destacar que além das melhorias fisiológicas, ela promove benefícios no âmbito social e mental, de modo que terá como resultados positivos a saúde (KWAG K., MARTIN P., RUSSELL D., FRANKE W., KOHUT M., 2011 citados por SOARES D.S.C., SOARES J.J., GRAUP S., STREB A.R., 2018).

Atualmente, sabe-se que a adoção de um estilo de vida ativo e a manutenção dele durante a gestação pode trazer benefícios não somente para a mãe, mas também para o bebê (ARTAL R, O'TOOLE M, 2003 citados por SOARES D.S.C., SOARES J.J., GRAUP S., STREB A.R., 2018). Esses benefícios são comparados a outro estudo realizado por (BATISTA et al 2003 citados por SILVA, G. BARROS, T. LISBOA, P. OLIVEIRA, 2017) que afirma que as atividades realizadas durante o período gestacional atingem várias áreas do organismo materno, trazendo desta forma proveitos como a redução e prevenção das lombalgias. Esses benefícios se dão devido a orientação postural correta da futura mãe frente a hiperlordose que comumente aparece durante o período gestacional, em função do crescimento do útero 
na cavidade abdominal e o consequente afastamento do centro gravitacional.

Portanto, com o auxílio de outros métodos, como a terapia nutricional e exercícios físicos, é muito útil para evitar complicações para mãe e filho (JACOB et al., 2014 citados por MENDES, MARTINS K., et al 2017). Existem alguns tipos de exercícios, onde a mulher pode aumentar a sua resistência, fortalecer os músculos do abdômen e outros fatores que irão ajudar durante a gravidez e o trabalho de parto.

De acordo com (LEITÃO et al. 2000 citados por MASCARENHAS et al. 2016) destacam que a prática do exercício físico para gestantes é de suma importância, pois essa prática ajuda para a manutenção da aptidão física e da saúde, diminui os sintomas gravídicos, melhora o controle ponderal, diminui a tensão no parto e contribui para a recuperação do mesmo. O presente estudo aborda a importância da atividade física praticada de maneira eficiente em gestantes com obesidade e diabetes, além de diminuir os riscos de doenças relacionadas, resultando em uma gestação mais tranquila, além de uma melhor recuperação pós-parto.

A atividade física apresenta resultados benéficos para gestantes com diabetes e obesidade? Diante do que foi introduzido a atividade física apresenta sim resultados satisfatórios no controle da diabetes e obesidade, sendo feita com acompanhamento e cuidados devidos. Portanto, o objetivo geral desse estudo foi avaliar através das evidências científicas os principais efeitos da atividade física durante a gestação, além de investigar através dos estudos encontrados, os principais efeitos no controle da diabetes e obesidade na gestação e descrever os principais benefícios obtidos através da prescrição de diferentes tipos de treinamentos em gestantes.

\section{METODOLOGIA |TIPO DE ESTUDO}

O presente trabalho foi realizado através de pesquisa bibliográfica que é caracterizado por parte da bibliografia publicada, seja ela em livros, revistas, publicações. Tal revisão de literatura tem como objetivo discutir sobre a Atividade física e os principais efeitos no controle da diabetes e obesidade na gestação, e os principais benefícios obtidos através na prescrição de diferentes tipos de atividade física em gestantes.

\section{COLETA DE DADOS}

Para a realização desta pesquisa foram realizados levantamentos bibliográficos de artigos científicos achados nas bases de dado Pubmed, The Scientific Electronic Library Online - (SCIELO). Os dados foram coletados no período de março a maio de 2020, onde foram selecionados alguns artigos por meio da associação dos seguintes descritores através da forma boleana "AND":

10 associação: Gestação AND atividade física;

2o associação: Obesidade AND diabetes; 


\section{METODOLOGIA |CRITÉRIOS DE INCLUSÃO E EX- CLUSÃO DE ESTUDOS}

AOs critérios de inclusão para o uso dos estudos serão artigos completos referentes à pesquisa ( $A$ atividade física e os principais efeitos no controle da diabetes e obesidade na gestação), idioma (português e inglês), tempo do artigo (artigos publicados nos últimos 10 anos, 2010 a 2020), tipo de estudo (artigos) e base de dados (SCIELO e Pubmed). Os critérios para exclusão dos estudos serão: teses, resumos, congressos e textos incompletos, artigos publicados a mais de 10 anos, artigos que fogem à temática e artigos duplicados.

\section{ANÁLISE DOS DADOS}

Os dados encontrados serão foram analisados, comparados e discutidos, permitindo, desta forma, a formulação de considerações importantes sobre o tema abordado neste trabalho. Finalizada a coleta de dados, será feita foi feita uma análise dos objetivos e resultados de todos os estudos a fim de obter os pontos de partida e desfechos que mais fundamentaram o tema pesquisado.

\section{RESULTADOS}

Nesta revisão de literatura foram incluídos 14 artigos. Nas bases de dados foram encontrados 22 artigos, destes, 16 foram na Pubmed, SCIELO 6. Excluiu-se 9 por não atenderem os critérios propostos: artigos completos referentes à pesquisa (Gestação and atividade física, obesidade and diabetes; idioma (português e inglês), tempo do artigo (artigos publicados nos últimos 05 anos, 2015 a 2020), tipo de estudo (artigos), base de dados (Pubmed e SCIELO) e artigos duplicados.

Na figura 1, através da associação Gestação and atividade física, obesidade and diabetes foram encontradas 8 referências na base de dados Pubmed, destes, 8 artigos foram publicadas na íntegra, sendo 03 de língua portuguesa e 05 inglesa, todos estavam no período de 2015 a 2020 com título condizente com os descritores, atendendo desta forma ao recorte temporal em estudo e a temática proposta. 
FIGURA 1. Representação gráfica das associações Gestação AND atividade física, obesidade AND diabetes encontradas na Pubmed.

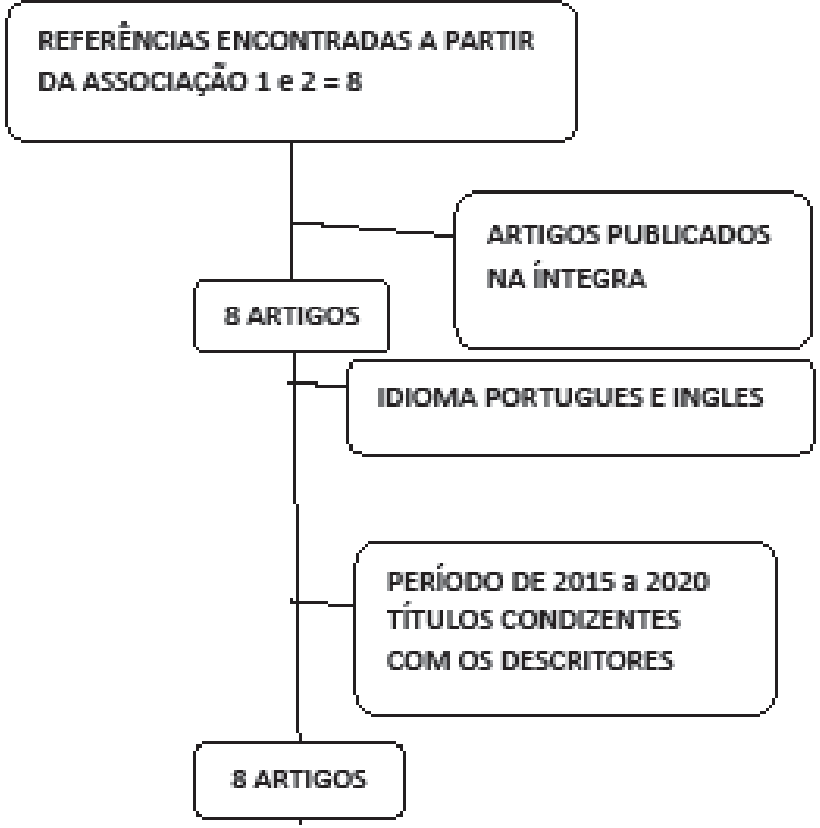

Fonte: Pesquisa realizada em bases de dados.

Na figura 2, através da associação Gestação and atividade física, obesidade and diabetes foram encontradas 5 referências na base de dados Scielo, destes, 6 artigos foram publicadas na íntegra, sendo 05 de língua portuguesa e 01 inglesa, todos estavam no período de 2015 a 2020 com título condizente com os descritores, atendendo desta forma ao recorte temporal em estudo e a temática proposta.

FIGURA 2. Representação gráfica das associações Gestação AND atividade física, obesidade AND diabetes encontradas na Scielo.

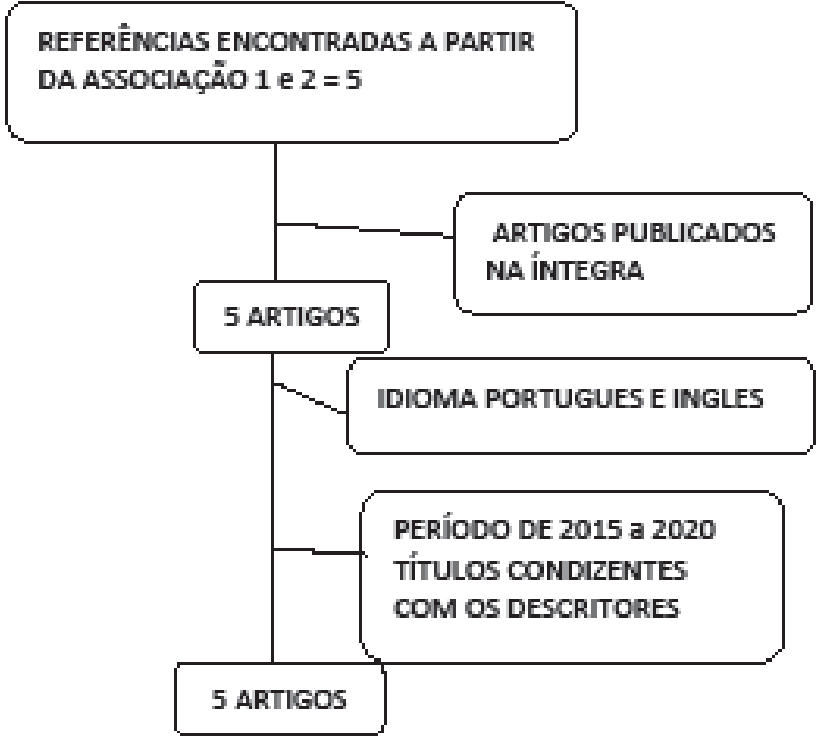

Fonte: Pesquisa realizada em bases de dados. 
A tabela 1 mostra que dos anos de publicação dos artigos, o ano de 2018 teve maior prevalência, com (38,46\%), seguido pelo ano de 2017 com uma porcentagem um pouco menor (30,77\%). Observam-se ainda o ano de 2019 teve uma menor quantidade de artigos publicados no ano $(07,69 \%)$, e o ano de 2016, também teve um resultado razoável (23,47\%), comparado ao de 2019. Comparado também a isso, o ano de 2015 e 2020 não tiveram número de artigos publicados. De acordo com a tabela percebe-se que o estudo obteve de um intervalo de tempo adequado acerca do tema, possibilitando um contraste e comparação dos anos.

Tabela 01: Distribuição dos estudos incluídos na amostra, referentes ao ano de publicação.

\begin{tabular}{|c|c|c|}
\hline ANO DE PUBLCAÇÃO & N ABSOLUTO & $\mathbf{\%}$ \\
\hline 2016 & 03 & $23,07 \%$ \\
\hline 2017 & 04 & $30,77 \%$ \\
\hline 2018 & 05 & $38,46 \%$ \\
\hline 2019 & 01 & $07,69 \%$ \\
\hline TOTAL & $\mathbf{1 3}$ & $\mathbf{1 0 0 \%}$ \\
\hline
\end{tabular}

Fonte: Pesquisa realizada em bases de dados.

Observa-se na tabela 2 que a fonte online PUBMED com 61,53\% da amostra, foi a que mais disponibilizou estudos sobre a temática proposta em relação às outras fontes online, seguido por SCIELO que apresentou $38,46 \%$.

TABELA 02 - Distribuição dos estudos incluídos na amostra referentes à fonte online de publicação.

\begin{tabular}{|c|c|c|}
\hline FONTE ONLINE & $\mathbf{N}^{\circ}$ ABSOLUTO & $\%$ \\
\hline SCIELO & 05 & $38,46 \%$ \\
\hline PUBMED & 08 & $61,53 \%$ \\
\hline TOTAL & $\mathbf{1 3}$ & $\mathbf{1 0 0} \%$ \\
\hline
\end{tabular}

Fonte: Pesquisa realizada em bases de dados.

Quanto aos periódicos, na tabela 3 observa-se que a Revista Eur J Obstet Gynecol Reprod Bio obteve maior número de artigos publicados, com 20\%, apesar de ser apenas uma revista a frente as demais, foi a que teve maior publicação. Um total de 2 revistas tiveram apenas um artigo utilizado no estudo cada uma, que somando contribuíram imensamente para o trabalho.

TABELA 3: Distribuição dos estudos incluídos na amostra, referentes ao nome do periódico.

\begin{tabular}{|l|c|c|}
\hline \multicolumn{1}{|c|}{ NOME DO PERIÓDICO } & N & ABSOLUTO \\
\hline Rev. EurJ ObstetGynecol Reprod Biol & 02 & $20 \%$ \\
\hline Rev. Par Enferm & 01 & $10 \%$ \\
\hline Rev. Eur J Epidemiol & 01 & $10 \%$ \\
\hline Rev. Glob Adv Health Med. & 01 & $10 \%$ \\
\hline Rev. Hormones (Athens, Greece) & 01 & $10 \%$ \\
\hline Rev. R. Perspect. Ci. e Saúde & 01 & $10 \%$ \\
\hline Rev. Edição Especial PIBIC & 01 & $10 \%$ \\
\hline Rev. Rev Enferm Atenção Saúde & 01 & $10 \%$ \\
\hline
\end{tabular}




\begin{tabular}{|r|c|c|}
\hline Rev. Revista Pró-UniverSUS & 01 & $10 \%$ \\
\hline Rev. Archives Of Health Investigation & 01 & $10 \%$ \\
\hline TOTAL & 10 & $\mathbf{1 0 0 \%}$ \\
\hline
\end{tabular}

Fonte: Pesquisa realizada em bases de dados.

O quadro 1 diz respeito aos autores das bases teóricas utilizados na pesquisa, de forma que se evidenciou que todos tratam-se de artigos científicos; o tema do assunto, bem como o ano de publicação referente a cada publicação utilizada na construção da pesquisa.

Quadro 1 - Distribuição dos autores, tipo de estudo, objetivo e resultados dos estudos:

\begin{tabular}{|c|c|c|c|c|}
\hline ARTIGO & $\begin{array}{l}\text { AUTORES/ } \\
\text { ANO }\end{array}$ & $\begin{array}{l}\text { TIPO DE } \\
\text { ESTUDO }\end{array}$ & $\begin{array}{l}\text { OBJETIVO DO } \\
\text { ESTUDO }\end{array}$ & RESULTADOS \\
\hline 1 & $\begin{array}{l}\text { Ruben } \\
\text { Barakata, } \\
\text { Evelia Fran- } \\
\text { co, María } \\
\text { Perales, Car- } \\
\text { mina López, } \\
\text { Michelle } \\
\text { F. Mottola, } \\
\text { (2018) }\end{array}$ & $\begin{array}{l}\text { Ensaio } \\
\text { clínico ran- } \\
\text { domizado }\end{array}$ & $\begin{array}{l}\text { Examinar a influên- } \\
\text { cia de um progra- } \\
\text { ma de exercícios } \\
\text { durante a gravidez } \\
\text { na duração do tra- } \\
\text { balho de parto em } \\
\text { mulheres grávidas } \\
\text { saudáveis. }\end{array}$ & $\begin{array}{l}\text { As mulheres randomizadas para o GE tiveram } \\
\text { menor primeira etapa do trabalho de parto, } \\
\text { bem como a duração combinada de primeira } \\
\text { e segunda etapas de trabalho de parto. }\end{array}$ \\
\hline 2 & $\begin{array}{l}\text { Gabriele Sac- } \\
\text { cone, Vincen- } \\
\text { zo Berghella, } \\
\text { Fulvio Zullo, } \\
\text { et al., (2018) }\end{array}$ & $\begin{array}{l}\text { Obser- } \\
\text { vacional } \\
\text { prospec- } \\
\text { tivo }\end{array}$ & $\begin{array}{l}\text { Avaliar os efeitos } \\
\text { do exercício duran- } \\
\text { te a gravidez em } \\
\text { gestações únicas } \\
\text { assintomáticas sem } \\
\text { parto prematuro } \\
\text { espontâneo prévio } \\
\text { (SPTB), mas com } \\
\text { comprimento cervi- } \\
\text { cal ultrassonográfico } \\
\text { transvaginal curto } \\
\text { (TVU CL). }\end{array}$ & $\begin{array}{l}\text { Análise secundária, as mulheres foram classifi- } \\
\text { cadas nospor classificação de grupos usando as } \\
\text { informações constadas de mês em mês, e tipos } \\
\text { de exercício. } \\
\text { exercícios < } 2 \text { dias por semana } 20 \text { minutos por dia. } \\
\text { O resultado primário de }\end{array}$ \\
\hline 3 & $\begin{array}{l}\text { Rebesco, De- } \\
\text { nis \& Souza, } \\
\text { William \& } \\
\text { Lima, Valderi } \\
\text { \& Grzelczak, } \\
\text { Marcos \& } \\
\text { Frasson, } \\
\text { Antonio \& } \\
\text { Mascare- } \\
\text { nhas, Luis. } \\
\text { (2016). }\end{array}$ & $\begin{array}{l}\text { Um estudo } \\
\text { de revisão. }\end{array}$ & $\begin{array}{l}\text { Sendo assim, este } \\
\text { estudo buscou } \\
\text { como objetivo reali- } \\
\text { zar uma revisão bi- } \\
\text { bliográfica e analisar } \\
\text { informações perti- } \\
\text { nentes sobre a ação } \\
\text { do exercício físico a } \\
\text { respeitos dos riscos } \\
\text { e benefícios durante } \\
\text { a gestação. }\end{array}$ & $\begin{array}{l}\text { As seguintes recomendações foram semelhantes } \\
\text { na maioria dos trabalhos que o exercício físico } \\
\text { contribui para manutenção da aptidão física, } \\
\text { diminui sintomas gravídicos, melhora o controle } \\
\text { ponderal, diminui a tensão no parto, ajuda na re- } \\
\text { cuperação no pós-parto, bem como prevenção de } \\
\text { algumas desordens, como diabetes gestacional. }\end{array}$ \\
\hline
\end{tabular}




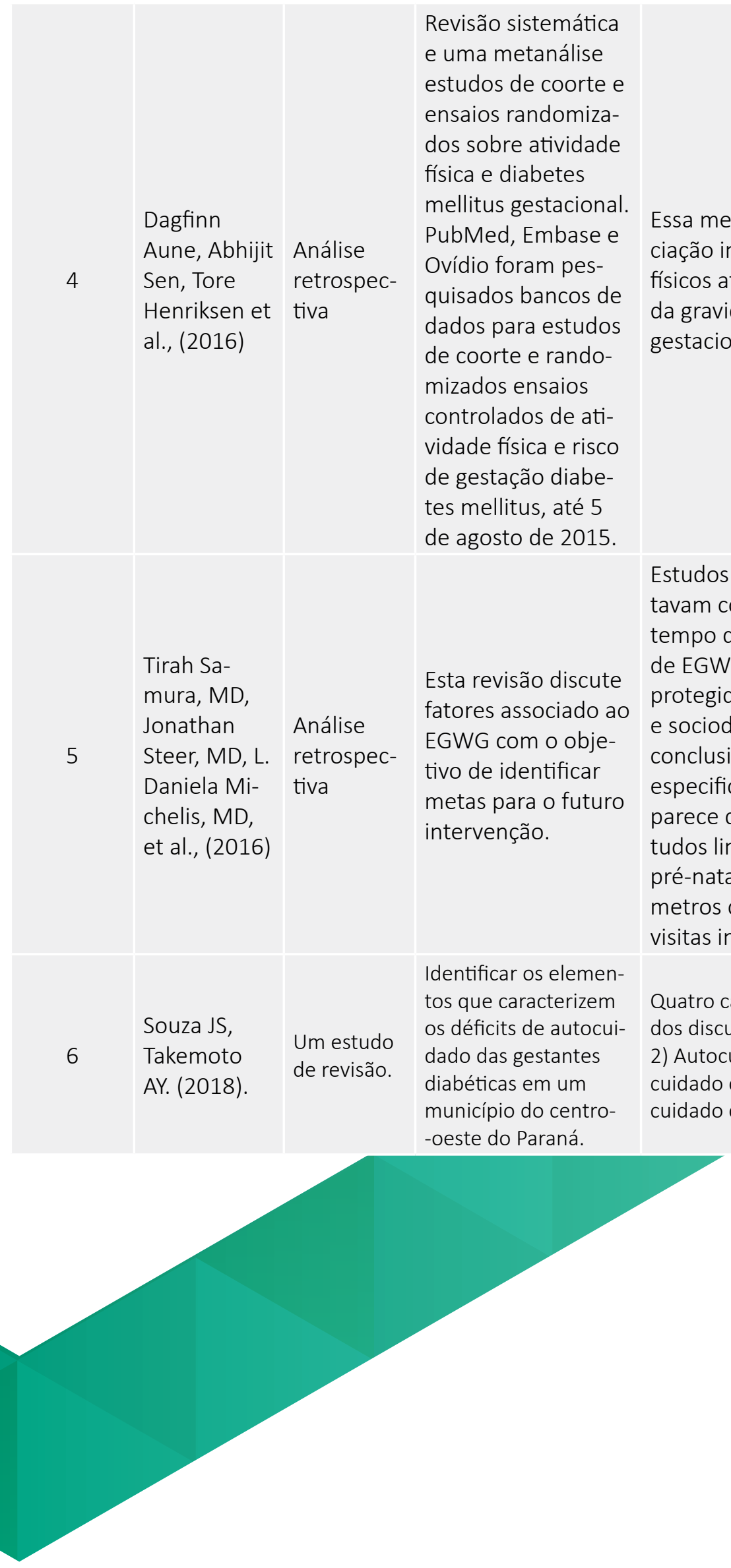

Revisão sistemática e uma metanálise estudos de coorte $e$ ensaios randomizados sobre atividade física e diabetes mellitus gestacional. PubMed, Embase e Ovídio foram pesquisados bancos de dados para estudos de coorte e randomizados ensaios controlados de atividade física e risco de gestação diabetes mellitus, até 5 de agosto de 2015.

Esta revisão discute fatores associado ao com o objede identificar metas para o futuro intervenção.

Identificar os elementos que caracterizem os déficits de autocuidado das gestantes abéticas em um município do centrooeste do Paraná.
Essa metanálise sugere que existe uma associação inversa significativa entre os fatores físicos atividade antes da gravidez e no início a gravidez e o risco de diabetes mellitus estacional.

Estudos descobriram que mulheres que escom sobrepeso ou obesidade em o po da concepção estava mais alto risco GWG e que aumentou atividade física rotegida contra EGWG. Estudos sobre dieta ciodemografia características foram inclusivo. Fatores psicológicos, percepções ecificamente precisas de IMC também parece desempenhar um papel EGWG. Esdos limitados sobre métodos assistência ré-natal não mostrar melhora de peso parâtros com o grupo em comparação com itas individuais

uatro categorias emergiram a partir da análise os discursos: 1) Autocuidado com a alimentação, com a atividade física e lazer e 4) Autoidado com as atividades de vida diárias. 
O presente estudo teve como objetivo realizar revisão sistemática nas bases de dados bibliográficas sobre a taxa metabólica Taousani E, Savvaki D, Revisão Tsirou $\mathrm{E}$, et sistemática al. (2017)

Observacio- basal (TMB), maior componente do GE, durante a gestação de feto único de mulheres saudáveis. Segundo os critérios de inclusão, 37 artigos foram selecionados (24 estudos de coorte e 13 seccionais).

Buscando melhor qualidade de vida, diminuição do estresse e prevenção de comorbidades, as práticas
O aumento da TMB (entre 8,0 e 35,0\%) ocorreu na maioria de estudos de coorte dependendo do tempo de seguimento e do estado nutricional. Nos seccionais, o aumento na TMB foi de $8,0-28,0 \%$ na fase final da gestação sobre a fase inicial ou no pós-parto. Informação precária sobre idade materna, perdas de seguimento e curto tempo de acompanhamento durante a gestação foram limitações dos estudos revisados. Em conclusão, a TMB aumenta durante a gestação e o aumento é mais intenso a partir do 20 trimestre. As estimativas mais confiáveis provêm dos poucos estudos de coorte iniciados na fase pré-gestacional.

Nesse sentido, os profissionais de saúde, principalmente os médicos que estão próximos das gestantes, são os responsáveis por disseminar essas concepções e orientar as mulheres sobre os benefícios da prática da atividade física durante a gestação. Essa orientação deve ser feita tanto para as muIheres fisicamente ativas quanto para aquelas sedentárias. No caso das sedentárias, a orientação é de atividades de intensidade leve a moderada, que podem ser ou não supervisionadas, na dependência das condições da gestante, e nesse caso a caminhada pode ser o início de uma proposta maior. Para as muIheres fisicamente ativas a orientação deve ser a manutenção das atividades físicas compatíveis com o período gestacional, e elas geralmente já desenvolvem alguma atividade supervisionada, o que torna mais fácil realizar mudanças sem perda dos benefícios cardiovasculares, entre outros. A orientação para a realização de atividade física durante a gestação é estimulada e indicada pelo American College of Obstetricians and Gynecologists (ACOG) desde a década de 1990. Porém, somente em 2002 essa prática foi reconhecida como segura e indicada para todas gestantes saudáveis. 

(2018).

Observacional prospectivo.

Observacio-

nal

Kunzendorff

BA, Nicoli

11

BM, Luz FA,

Martins KG,

Mendes AA.

(2017)

Brandão, PZ;

12

da Silva, TB; Pesquisa

de Siqueira, exploratória

EC. (2019)

Queiroga

MG, Car-

13
O presente estudo

teve como objetivo

analisar a importância

da prática de ativi-

dade física durante a

gestação e lactação.

Este estudo tem como objetivo relatar a experiência de acadêmicos de enfermagem com gestantes no pré-natal sobre a prevenção da obesidade como fator de risco para a gravidez em uma unidade de referência em Belém.

Sendo assim, o objetivo deste trabalho é apresentar as possíveis causas do desenvolvimento desta doença, além da fisiopatologia, prováveis complicações e consequências no período pré e pós-parto.

Este artigo visa a construção de uma pesquisa exploratória que utiliza como coleta de dados o levantamento bibliográfico de artigos científicos e livros da área examinada.

Verificar a adesão à atividade física em grávidas.
Foi identificado que apesar de não haver estudos decisivos em relação ao padrão ótimo de atividade física e exercícios durantes o período da gestação há um consenso na literatura de que a atividade física regular e contínua, realizada com intensidade de leve a moderada proporciona vários benefícios e, consequentemente, diminuição de riscos e complicações durante a gestação.

Os resultados obtidos durante a oficina "Cuide-se! Obesidade como fator de risco" evidenciam pouco conhecimento das gestantes, depoimentos e perguntas sobre o tema, assim como a satisfação das mesmas por terem tirado suas dúvidas sobre a importância da prevenção. O objetivo do estudo foi alcançado ao relatar a vivência de acadêmicos de enfermagem na prevenção da obesidade na gestação por meio de uma ação educativa.

Sendo assim, de acordo com a análise e seleção de artigos relacionados à Diabetes na gestação, conclui-se que esta é uma patologia comum e que há diversos meios para prevenção e tratamento. Além disso, as complicações materno fetais também são abordadas e associadas às formas de se evitar tais problemas. A presença de uma equipe interdisciplinar e um acompanhamento pré-natal eficiente também aumentam as probabilidades de sucesso na gestação.

Assim, a obesidade associa-se de maneira direta ao prognóstico da gestação. Desta maneira, a perda de peso, prática regular de atividades físicas e alimentação saudável devem ser estimuladas pelos profissionais de saúde, com o fim de promover a saúde materna e fetal.

Sugere-se que sejam feitas mais pesquisas como essa, mas com gestantes fora do sistema público para comparação e para que possa se discutir ainda mais sobre o quão importante é que seja mudado esse cenário que apresentado nesse estudo, pois as realizações das atividades regulares podem trazer mais benefícios do que riscos para as gestantes. 


\section{DISCUSSÃO}

A prática regular de atividade física, diminui diversos riscos para saúde, além de vários benefícios, como ajuda no controle do peso corporal, contribui para ossos e articulações e proporciona melhor qualidade de vida, para gestantes. Exercícios como a musculação também podem ser realizados durante a gravidez, sempre utilizando cargas leves e com a supervisão de um profissional de Educação Física o exercício de musculação melhora a postura da gestante e ajudar a reduzir o seu peso gestacional. (SOARES et al, 2017). A atividade física na gestação traz diversas melhorias para a saúde, o que é essencial para ter uma gravidez, pois controla o peso da gestante, ajuda na diabetes gestacional, além de proporcionar bem-estar e melhorar a autoestima. A atividade física também tem total importância no combate ao sedentarismo, não se faz necessário práticas com altos níveis de intensidade, o importante é a rotina de atividades para combater o sedentarismo e seus riscos. Conforme mencionado por (MONTENEGRO 2014 citado por SILVA, G. BARROS, T. LISBOA, P. OLIVEIRA, 2017), podemos observar que os benefícios com prática de atividade física neste período são grandes, pois melhora o nível de saúde da gestante.

Existem poucos estudos avaliando as repercussões da prática da atividade física na gestação, porém em um dos artigos foi feita uma pesquisa educativa, com algumas gestantes e acompanhantes. Foi abordado vários assuntos neste estudo, como hábitos, comorbidades, doenças crônicas. A abordagem da temática ocorreu por meio de uma dinâmica e contribuiu para que elas participassem ativamente. Alguns resultados pertinentes ao tema discutido foram retirados dessa pesquisa de acordo com nível de instrução de cada uma, e suas comorbidades, como se observa a seguir. Em estudo realizado com 255 mulheres grávidas diabéticas 61,9\% destas teve via de parto cirúrgica o que suscita maiores cuidados e controle quanto ao nível glicêmico na gestação. A diabetes é uma das principais complicações tardias associadas a obesidade gestacional, com risco de $30 \%$ das mulheres desenvolverem Diabete Mellitus 2 em 5 a 16 anos após o parto, sobretudo se continuarem obesas, mulheres que excedem a ingestão calórica recomendada no decorrer da gravidez estão seriamente suscetíveis ao ganho de peso exacerbado com progressão para a obesidade, concomitante a morbidades como hipertensão arterial e diabetes, os quais podem persistir após o parto. É importante que o profissional de saúde informe a correlação entre obesidade adquirida na gestação com as condições de saúde futuras da criança. Esses resultados demonstram dúvidas relevantes por parte das gestantes, indicando pouco conhecimento acerca de diferentes aspectos que envolvem o período de gestação, principalmente quando relaciona isso a doenças que já existem antes do período gestacional.

É recomendado que as mulheres com excesso de peso recebam orientações nutricionais desde o período pré-gestacional até o período pós-gestacional, com intuito de reduzir as complicações perinatais. Assim, a alimentação saudável, a prática regular de atividades físicas e a normalização do peso são fatores que devem ser estimulados não só durante a gravidez, mas de forma de permanente. Segundo (SILVA, 2012 citado por CARMO, A. P., 2018) exercícios resistidos com pesos de intensidade leve a moderada podem promover melhora na resistência e flexibilidade muscular, sem aumento no risco de lesões, de complicações na gestação ou relacionados ao peso do feto ao nascer. Além desses exercícios prevenir, e controlar comorbidades, como obesidade e diabetes.

A prática de atividade física ajuda na recuperação da mulher durante o período pós-parto e na gravidez foi identificado que mulheres que não praticam exercícios físicos engordam mais que outras gestantes praticantes de atividade física. Isso pode causar um risco maior para seu bebê durante a gravidez e com uma recuperação mais lenta (FERREIRA et al, 2014). 


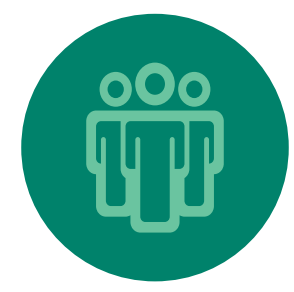

\section{CONCLUSÃO}

Os resultados vistos no estudo relatam que gestantes com comorbidades, como diabetes e obesidade que fizeram exercícios físicos com acompanhamento dentro dos níveis de intensidade indicado, tiveram êxitos que não comprometeram o período gestacional nem o feto.

Existem algumas modalidades de exercício que são mais apropriadas ao período da gravidez, aqueles de baixo impacto e riscos. A escolha da mesma passa por fatores como as próprias comorbidades, idade, tempo de gestação.

Portanto a atividade física durante a gestação traz inúmeros benefícios para a mãe e para o feto, desde que sejam tomados os devidos cuidados quanto ao tipo, duração e intensidade dos exercícios, respeitando as contraindicações e patologias associadas com acompanhamento profissional e indicação médica de maneira individualizada.

Para tanto, constatou-se que a atividade física acompanhada por profissionais da saúde durante a gestação é muito importante, para a prevenção de doenças durante e após o período gestacional ajudando no crescimento do bebê e na recuperação mais rápida da gestante. 


\section{REFERÊNCIAS}

AUNE D, SEN A, HENRIKSEN T, SAUGSTAD OD, TONSTAD S. Physical activity and the risk of gestational diabetes mellitus: a systematic review and dose-response meta-analysis of epidemiological studies. Eur J Epidemiol. 2016 Oct;31(10):967-997. doi: 10.1007/s10654-016-0176-0. Epub 2016 Aug 2. PMID: 27485519; PMCID: PMC5065594

BRANDÃO, PZ; DA SILVA, TB; DE SIQUEIRA, EC. Obesidade e gestação: a importância da correlação na avaliação dos riscos materno-fetais. Revista Pró-UniverSUS. 2019 Jul./Dez.; 10 (2): 18-23

CARMO, A. P. (2018). Benefícios da atividade física na gestação e lactação. CENTRO ACADÊMICO DE VITÓRIA DE SANTO ANTÃO NÚCLEO DE EDUCAÇÃO FÍSICA E CIÊNCIA DO ESPORTE.

KUNZENDORFF BA, NICOLI BM, LUZ FA, MARTINS KG, MENDES AA. A influência da diabetes mellitus no período gestacional como fator de risco. III Seminário Científico da FACIG - 09 e 10 de Novembro de 2017 II Jornada de Iniciação Científica da FACIG - 09 e 10 de Novembro de 2017.

QUEIROGA MG, CARRIEL MGL, PERUCHI LH. Prática de atividade física durante a gestação. Edição Especial PIBIC, outubro 2018 ISSN 2525-5250

REBESCO, DENIS \& SOUZA, WILLIAM \& LIMA, VALDERI \& GRZELCZAK, MAROCS \& FRASSON, ANTONIO \& MASCARENHAS, LUIZ. (2016). Ação do exercício físico na gestação: Um estudo de revisão. ARCHIVES OF HEALTH INVESTIGATION. 5. 10.21270/archi.v5i6.1707.

RUBEN BARAKAT, EVELIA FRANCO, MARIA PERALES, CARCMINHA LOPEZ, MICHELLE F. MOTOLLA. Exercise during pregnancy is associated with a shorter duration of labor. A randomized clinical trial. Eur J Obstet Gynecol Reprod Biol. 2018 Mar 6; 224: 33-40. Published online 2018 Mar 6. doi: 10.1016/j. ejogrb.2018.03.009

SAMURA T, STEER J, MICHELIS LD, CARROL L, HOLLAND E, PERKINS R. Factors Associated With Excessive Gestational Weight Gain: Review of Current Literature. Glob Adv Health Med. 2016 Jan;5(1):87-93. doi: 10.7453/gahmj.2015.094. Epub 2016 Jan 1. PMID: 26937318; PMCID: PMC4756783.

SILVA A.I.S., Freitas I.A., Almeida L.S., Oliveira M.N.S., Rocha P.S.S., Matos E.C.O. A obesidade na gestação através e ações educativas em saúde. Rev Enferm Atenção Saúde [Online]. Jul/Dez 2017; 6(2):144152

SILVA, P. E. C.; LISBOA, T. S.; BARROS, G. DOS S.; OLIVEIRA, P. S. P. DE. Musculação para gestantes: entre efeitos e indicações. Revista Valore, v. 2, n. 1, p. 186-194, 2 ago. 2017.

SOARES D.S.C., SOARES J.J., GRAUP S., STREB A.R. Atividade física na gestação: uma revisão integrativa. R. Perspect. Ci. e Saúde 2017;2(2): 71-84.

SOUZA J.S., TAMKEMOTO A.Y (2018). Diabetes mellitus gestacional e as dificuldades para o autocuidado. REPENF - Rev Par Enferm. 2018; 1(1): 37-46.

TAOUSANI E, SAVVAKI D, TSIROU E et al. Regulation of basal metabolic rate in uncomplicated pregnancy and in gestational diabetes mellitus. Hormones (Athens, Greece). 2017 Jul;16(3):235-250. DOI: 10.14310/horm.2002.1743. 